19 Revue d'histoire du XIXe siècle

Société d'histoire de la révolution de 1848 et des

révolutions du XIXe siècle

$24 \mid 2002$

Varia

Jeremy D. POPKIN, Press, Revolution, and Social

Identities in France, 1830-1835

University Park, The Pennsylvania State University Press, 2002, X--329 p.

Thomas Bouchet

\title{
OpenEdition
}

Journals

Édition électronique

URL : http://journals.openedition.org/rh19/387

ISSN : 1777-5329

Éditeur

La Société de 1848

Édition imprimée

Date de publication : 1 juin 2002

Pagination : 186-188

ISSN : 1265-1354

Référence électronique

Thomas Bouchet, « Jeremy D. POPKIN, Press, Revolution, and Social Identities in France, 1830-1835»,

Revue d'histoire du XIXe siècle [En ligne], 24 | 2002, mis en ligne le 28 juin 2005, consulté le 21

septembre 2020. URL : http://journals.openedition.org/rh19/387

Ce document a été généré automatiquement le 21 septembre 2020.

Tous droits réservés 


\section{Jeremy D. POPKIN, Press, Revolution, and Social Identities in France,}

\section{0-1835}

University Park, The Pennsylvania State University Press, 2002, X--329 p.

\section{Thomas Bouchet}

La recherche dont Jeremy Popkin livre ici les résultats porte conjointement sur trois objets d'histoire qu'il n'est pas fréquent d'articuler avec autant de netteté. Histoire de la presse, histoire des révolutions, histoire des identités sociales sont certes liées par mille fils. Mais l'originalité du livre réside dans le projet de constitution d'un champ d'étude spécifique à partir de ces trois composantes, dont la presse est l'élément clé. Depuis son Revolutionary News: The Press in France 1789-1799 (Duke University Press, 1990), l'approche de J. Popkin s'est ainsi à la fois un peu déplacée et enrichie de préoccupations nouvelles, d'autant que sa réflexion s'attache aussi à des questions qui forment comme l'arrière-plan de son projet central : dans une introduction riche et dense, puis au fil des pages, J. Popkin se situe par rapport à certaines interrogations d'histoire culturelle: selon lui, l'importance des journaux "ne tiendrait pas essentiellement à leur capacité à fournir information et idéologie --même s'il convient de ne pas mésestimer ce rôle-- mais à leur place dans la construction d'identités sociales et culturelles, ainsi qu'à leur statut littéraire". (p.9). De même, la notion d'événement fait l'objet de la plus grande attention. L'événement révolutionnaire est ici conçu comme point de cristallisation majeur, dont les logiques et les dynamiques tiennent en particulier au travail d'interprétation fourni par la presse : le chapitre $6 \mathrm{du}$ livre ("Textualizing insurrection", pp. 193-228) présente les journaux lyonnais "dans le feu de l'action", œuvrant chacun pour gagner le droit de donner sens à ce qui se produit. Le thème est familier à J. Popkin, qui a signé il y a deux ans un article préparatoire intitulé "La presse et les insurrections de Lyon en 1786 et 1831" ${ }^{1}$.

2 Press, Revolution, and Social Identities in France, 1830-1835 n'a pas cependant pour objectif de proposer une synthèse sur la question : le titre de l'ouvrage, beaucoup trop général, ne laisse pas entrevoir que l'auteur s'en tient modestement à une "étude de cas" 
(introduction, p. 22). Car son analyse porte sur Lyon. À la suite d'une série de chercheurs (dont Fernand Rude, Robert Bezucha et plus récemment Michèle RiotSarcey, souvent cités), il contribue à l'approfondissement de nos connaissances sur le Lyon des années 1830, de telle sorte que peu de villes françaises ont été aussi finement explorées par les historiens du social et du politique. Les archives exploitées par J. Popkin sont la plupart du temps bien connues, qu'il s'agisse des sources manuscrites ou d'une presse très diverse et d'une extrême vitalité (de l'Écho de la fabrique au Courrier de Lyon, du Précurseur au Conseiller des femmes). L'auteur se penche aussi longuement sur l'ensemble de l'œuvre de Jean-Baptiste Monfalcon (notamment l'Histoire des insurrections de Lyon --1834-- et les Souvenirs d'un bibliothécaire --1853) et sur un roman publié anonymement en 1835 et intitulé La révolte de Lyon en 1834, ou la fille du prolétaire.

Le livre est construit en quatre temps. Le premier chapitre est une mise en place générale qui présente "journaux, journalistes et espace public" à Lyon après 1830 ; les chapitres 2 à 4 portent sur les différents types de journaux lyonnais, en fonction de leur identité et de leurs orientations ("presse libérale ou bourgeoise"; "presse alternative", "presse des travailleurs"); c'est de banquets et de procès liés à la presse (chapitre 5) puis des liens entre presse et insurrections (chapitre 6) qu'il est question ensuite; le dernier chapitre est une étude sur le glissement qui s'opère "du journal au livre" dans l'écriture de l'événement révolutionnaire. Ce travail précis et rigoureux offre une masse très appréciable d'éléments sur les logiques de la vie sociale et culturelle lyonnaise au cours de quelques années cruciales. Logiquement, l'accent porte surtout sur les journaux et les journalistes (Marius Chastaing ou Anselme Petetin, par exemple); de nombreux éléments d'appréciation sont également fournis sur Monfalcon, personnalité si marquante dont l'action et l'œuvre dépassent largement le cadre de la presse, sur le préfet Adrien de Gasparin, ou encore sur Eugénie Niboyet. Le résultat est très stimulant et incite effectivement à entreprendre d'autres études de cas comparables. Ainsi serait-il possible de confirmer la solidité d'ensemble de la réflexion, de s'interroger aussi plus avant sur certaines hypothèses avancées par J. Popkin, quitte à les nuancer: faut-il considérer avec lui que "Lyon, entre 1830 et 1835, fut un microcosme où tous les courants politiques et sociaux de cette période nouvelle trouvèrent une claire expression" (p.264) alors que tant de travaux (le Ballots and Barricades de R. Aminzade, publié en 1993, en fournit un bon exemple) dégagent d'intéressantes spécificités selon les villes étudiées, qui aident à relativiser le cas lyonnais ? Est-il nécessaire, comme il le suggère p. 261, d'établir une ligne de partage si stricte entre le "champ [social, politique et culturel] polyphonique" dans lequel s'insère la presse, et la rigidité de livres d'histoire et de romans "statiques, figés, incapables de répondre aux événements qui surviennent" après publication, et ce malgré le jeu classique de réceptions et de réinterprétations auxquelles ils donnent lieu? Et, plus généralement: jusqu'à quel point la presse contribue-t-elle à forger les identités sociales et culturelles des Lyonnais? Nul doute qu'elle joue un rôle notable. Pourtant, lorsque Berger, le gérant de l'Écho de la fabrique, annonce à "la caste prolétaire" le 9 septembre 1832 (en première page) que "la presse est aujourd'hui une puissance supérieure à toutes les forces physiques, [qu'elle leur offre] son égide tutélaire [et que] de cette manière, se formera l'association universelle des travailleurs. [...]", la prudence impose de ne pas prendre la proclamation pour argent comptant, et d'y lire plutôt l'expression d'un ardent désir. 


\section{NOTES}

1. Jeremy D. POPKIN, “La presse et les insurrections de Lyon en 1786 et 1831", dans Hans-Jürgen LÜSEBRINK et Jean-Yves MOLLIER [dir.], Presse et événement : journaux, gazettes, almanachs (XVIII-XIXe siècles). Actes du colloque international "La perception de l'événement dans la presse de langue allemande et française”, Université de la Sarre, 12-14 mars 1998, Berne/Berlin/Bruxelles, Peter Lang, 2000. 